\title{
Ten-year experience with surgical unroofing of anomalous aortic origin of a coronary artery from the opposite sinus with an interarterial course
}

\author{
Peter C. Frommelt, MD, FACC, ${ }^{\text {a }}$ David C. Sheridan, MD, ${ }^{\text {a }}$ Stuart Berger, MD, ${ }^{\text {a }}$ \\ Michele A. Frommelt, MD, FACC, ${ }^{a}$ and James S. Tweddell, MD $^{\mathrm{b}}$
}

\begin{abstract}
Background: Anomalous aortic origin of a coronary artery from the opposite sinus with an intramural course between the great arteries (AAOCA) is associated with ischemia and sudden cardiac death in children, and surgical unroofing has been used to alleviate that risk.
\end{abstract}

\begin{abstract}
Methods: The cardiology database was reviewed to identify all patients with AAOCA who underwent surgical unroofing 10 years.
\end{abstract}

\begin{abstract}
Results: From March 1999 to September 2009, 27 patients with a mean age of $12.6 \pm 3.5$ years (range, 4-20 years) had surgical unroofing of AAOCA of the left coronary from the right sinus (left AAOCA, 7/27, 26\%) or of the right coronary from the left sinus (right AAOCA, 20/27, 74\%). Of these, 26 had diagnoses made by transthoracic echocardiography. Symptoms included resuscitated sudden cardiac death in 3, syncope in 8 , and chest pain in 4. No symptoms were present in 12 cases of serendipitous diagnosis. Unroofing of the intramural portion was successfully performed in all cases. A slitlike coronary orifice was described at surgical inspection in 12 patients, 7 of whom had right AAOCA and no symptoms. All patients after unroofing have patent coronary flow by Doppler and normal echocardiography and exercise treadmill testing at mean follow-up of 1.8 years. None have activity restrictions.
\end{abstract}

Conclusions: AAOCA is frequently characterized by an intramural course, which can be reliably identified by echocardiography. This form can be safely repaired by unroofing the intramural segment without early morbidity. Symptoms of possible ischemia are common but not always correlated with coronary ostial findings at surgery. (J Thorac Cardiovasc Surg 2011;142:1046-51)

Anomalous aortic origin of a coronary artery from the opposite sinus with an interarterial course between the great arteries (AAOCA) is often characterized by an intramural course of the anomalous coronary artery. This form of anomalous coronary origin has 2 subtypes, depending on which coronary originates from the wrong sinus: (1) anomalous left coronary from the right sinus of Valsalva (left AAOCA) and (2) anomalous right coronary from the left sinus (right AAOCA). The intramural segment in both forms carries coronary flow from the ostial opening in the wrong sinus within the anterior aortic wall until it exits the aorta from the appropriate sinus. Both right and left AAOCA are associated with myocardial ischemia and sudden cardiac death (SCD), particularly in adolescents and young adults, ${ }^{1-5}$ and interest in this lesion has grown during the past decade as multiple reports

From the Division of Pediatric Cardiology, ${ }^{\mathrm{a}}$ Department of Pediatrics, and the Department of Cardiothoracic Surgery, ${ }^{b}$ Children's Hospital of Wisconsin, Medical College of Wisconsin, Milwaukee, Wis.

Disclosures: Authors have nothing to disclose with regard to commercial support.

Received for publication Nov 5, 2010; revisions received Jan 16, 2011; accepted for publication Feb 9, 2011; available ahead of print March 25, 2011.

Address for reprints: Peter C. Frommelt, MD, FACC, Children's Hospital of Wisconsin, 9000 W Wisconsin Ave, Milwaukee, WI 53226 (E-mail: pfrom@mcw.edu). 0022-5223/\$36.00

Copyright (c) 2011 by The American Association for Thoracic Surgery doi:10.1016/j.jtcvs.2011.02.004 have documented successful identification of this anomaly by transthoracic echocardiography (TTE).$^{6-9}$ In addition, this form of AAOCA is amenable to repair by simply unroofing the intramural segment to create a neoorifice in the appropriate sinus without bypass grafting, coronary reimplantation, or patch angioplasty. ${ }^{9-11}$ In this report, we discuss the surgical experience at Children's Hospital of Wisconsin with intramural AAOCA, focusing on presentation, diagnosis, surgical findings, and early outcome.

\section{MATERIALS AND METHODS}

After approval was obtained from the institutional review board at Children's Hospital of Wisconsin, the cardiology database was reviewed for patients with the diagnosis of AAOCA who had undergone surgical unroofing of the intramural segment of the anomalous coronary within the anterior aortic wall. From this patient population, medical records, imaging studies, provocative testing to elicit myocardial ischemia, and surgical reports were reviewed to identify patients with AAOCA.

\section{RESULTS \\ Patient Population}

From September 1999 through September 2009, a total of 27 patients were noted to have an intramural AAOCA and underwent surgical unroofing. All patients were children or adolescents, with a median age at surgery of 13 years and a range of 4 to 20 years (Table 1). There were 


\section{Abbreviations and Acronyms}

$$
\begin{aligned}
\text { AAOCA = } & \text { anomalous aortic origin of a coronary } \\
& \text { artery } \\
\mathrm{SCD}= & \text { sudden cardiac death } \\
\mathrm{TTE}= & \text { transthoracic echocardiography }
\end{aligned}
$$

17 male and 10 female patients. Right AAOCA was identified in 20 patients and left AAOCA in 7 patients.

\section{Patient Presentation}

Symptoms raising suspicion of cardiac ischemia (syncope, chest pain, or aborted SCD) were present in 15 of the 27 patients and resulted in cardiologic evaluation that led to the diagnosis of an anomalous coronary artery (Figure 1). The 3 patients who had aborted SCD had left
AAOCA, and all episodes occurred during exercise. All were in medically unstable condition after presentation to the intensive care unit after resuscitation. Cardiopulmonary support with extracorporeal membrane oxygenation was necessary in 2 of these 3 patients because of persistent ventricular ectopy or low cardiac output after initial stabilization, despite easily identifiable laminar flow into the anomalous coronary by color Doppler. All 3 patients were taken to the operating room on an emergency basis for surgical unroofing because of ongoing evidence of myocardial dysfunction. All dysfunction resolved after surgery.

The other 12 patients underwent TTE for suspected congenital heart disease and were serendipitously found to have AAOCA. Four patients were found to have additional congenital heart disease; 2 had a perimembranous ventricular septal defect, which was closed at the time of surgical unroof-

\begin{tabular}{|c|c|c|c|c|c|c|c|c|c|}
\hline \multirow[b]{2}{*}{ Case } & \multirow[b]{2}{*}{ Age (y) } & \multirow[b]{2}{*}{ Sex } & \multirow[b]{2}{*}{ AOCA } & \multirow[b]{2}{*}{ Symptoms } & \multicolumn{2}{|c|}{ Imaging } & \multirow{2}{*}{$\begin{array}{l}\text { Associated } \\
\text { CHD }\end{array}$} & \multirow{2}{*}{$\begin{array}{c}\text { Ostial } \\
\text { stenosis }\end{array}$} & \multirow{2}{*}{$\begin{array}{c}\text { Surgical } \\
\text { technique }\end{array}$} \\
\hline & & & & & Dx & Additional & & & \\
\hline 1 & 13 & $\mathrm{~F}$ & LCA & Syncope with exercise & TTE & Cath & None & Yes & Unroofing \\
\hline 2 & 16 & M & LCA & None & TTE & Cath & Bicuspid AoV & No & Unroofing \\
\hline 3 & 11 & M & RCA & None & TTE & & VSD & Yes & $\begin{array}{l}\text { Unroofing; AoV resusp; } \\
\text { VSD closure }\end{array}$ \\
\hline 4 & 16 & M & LCA & SCD with exercise & TTE & TEE & None & No & Unroofing; AoV resusp \\
\hline 5 & 20 & M & LCA & SCD with exercise & Cath & & None & No & Unroofing; AoV resusp \\
\hline 6 & 4 & $\mathrm{~F}$ & RCA & None & TTE & Cath & VSD & No & $\begin{array}{l}\text { Unroofing; AoV resusp; } \\
\text { VSD closure }\end{array}$ \\
\hline 7 & 10 & M & $\mathrm{RCA}$ & Nonexercise $\mathrm{CP}$ & TTE & Cath & None & Yes & Unroofing \\
\hline 8 & 14 & M & RCA & $\mathrm{CP}$ with exercise & TTE & Cath & None & Yes & Unroofing \\
\hline 9 & 13 & M & RCA & None (benign PVCs) & TTE & & None & No & Unroofing \\
\hline 10 & 7 & M & $\mathrm{RCA}$ & None (heart murmur) & TTE & & None & Yes & Unroofing \\
\hline 11 & 16 & M & $\mathrm{RCA}$ & $\mathrm{CP}$ with exercise & TTE & & None & Yes & Unroofing \\
\hline 12 & 13 & $\mathrm{~F}$ & $\mathrm{RCA}$ & Nonexercise syncope & TTE & & None & Yes & Unroofing \\
\hline 13 & 9 & M & LCA & None (heart murmur) & TTE & & None & No & Unroofing \\
\hline 14 & 13 & $\mathrm{~F}$ & RCA & Syncope/CP with exercise & TTE & MRI & None & Yes & Unroofing \\
\hline 15 & 15 & $\mathrm{~F}$ & RCA & None (heart murmur) & TTE & & None & No & Unroofing \\
\hline 16 & 16 & M & RCA & Syncope with exercise & TTE & & None & No & Unroofing \\
\hline 17 & 13 & $\mathrm{~F}$ & $\mathrm{RCA}$ & Sleep apnea & TTE & & None & No & Unroofing \\
\hline 18 & 14 & $\mathrm{~F}$ & RCA & None & TTE & & Bicuspid AoV & Yes & Unroofing \\
\hline 19 & 16 & M & RCA & Nonexercise $\mathrm{CP}$ & TTE & & None & Yes & Unroofing \\
\hline 20 & 16 & M & RCA & Syncope with exercise & TTE & TEE & None & No & Unroofing \\
\hline 21 & 10 & $\mathrm{~F}$ & RCA & Syncope after exercise & TTE & $\mathrm{CT}$ & None & No & Unroofing \\
\hline 22 & 9 & M & RCA & Syncope with exercise & TTE & Cath & None & Yes & Unroofing \\
\hline 23 & 7 & $\mathrm{~F}$ & RCA & None (heart murmur) & TTE & $\mathrm{CT}$ & None & Yes & Unroofing \\
\hline 24 & 11 & M & LCA & None (abnormal ECG for LVH) & TTE & & None & No & Unroofing \\
\hline 25 & 15 & M & $\mathrm{RCA}$ & None (SVT) & TTE & & None & No & Unroofing \\
\hline 26 & 14 & M & LCA & SCD with exercise & TTE & & None & No & Unroofing \\
\hline 27 & 11 & $\mathrm{~F}$ & RCA & Syncope with exercise & TTE & & None & No & Unroofing \\
\hline
\end{tabular}
ing, and 2 had a bicuspid aortic valve that has not required intervention at latest follow-up. The remaining 8 patients had

TABLE 1. Clinical summaries

AOCA, Anomalous origin of coronary artery; $D x$, diagnostic; $C H D$, congenital heart disease; $F$, female; LCA, left coronary artery; TTE, transthoracic echocardiogram; Cath, cardiac catheterization; $M$, male; $A o V$, aortic valve; $R C A$, right coronary artery; $V S D$, ventricular septal defect; resusp, resuspension; $S C D$, sudden cardiac death; TEE, transesophageal echocardiography; $C P$, chest pain; $P V C$, premature ventricular contraction; $M R I$, magnetic resonance imaging; $C T$, computed tomography; $E C G$, electrocardiogram; $L V H$, left ventricular hypertrophy; $S V T$, supraventricular tachycardia. 


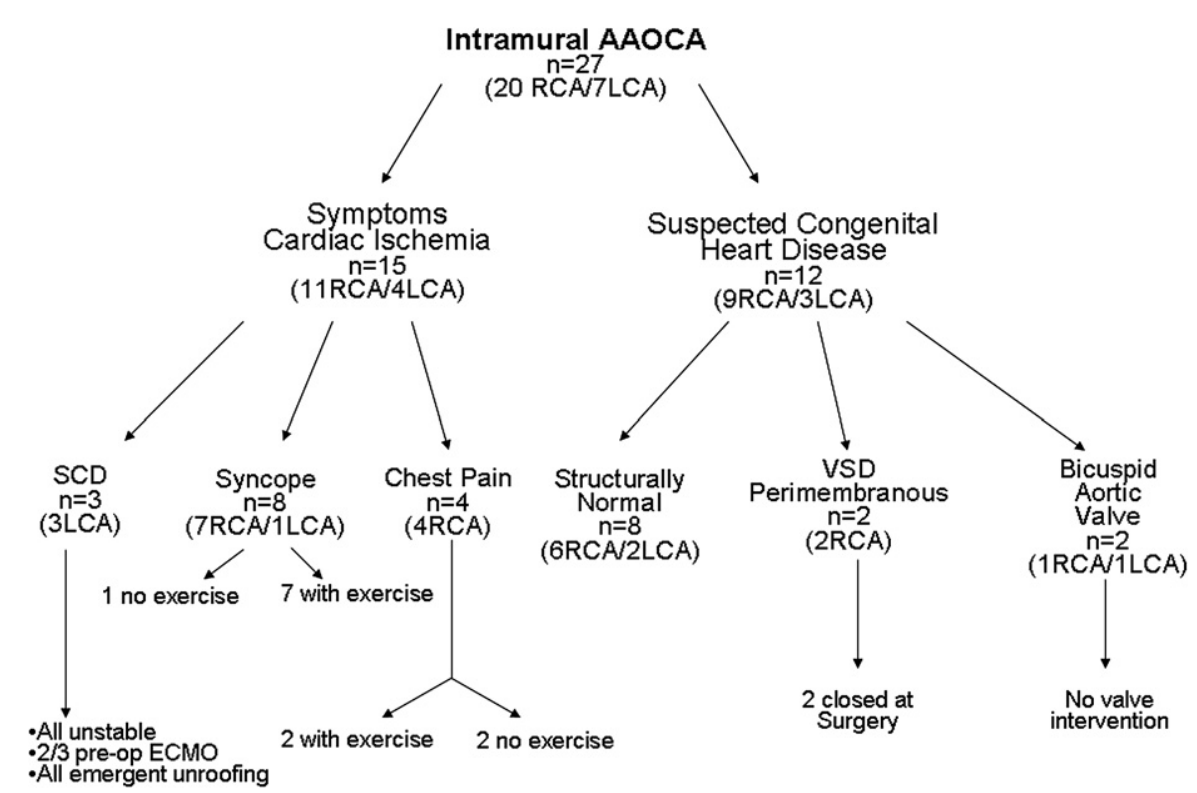

FIGURE 1. Intramural anomalous aortic origin of a coronary artery (AAOCA) outcomes. Flowchart details the rationale for evaluation and presentation symptoms in 27 patients with intramural anomalous aortic origin of a coronary artery at Children's Hospital of Wisconsin. The anomalous coronary is described in each subgroup as right coronary artery $(R C A)$ when the anomalous coronary was the right coronary from the left sinus of Valsalva and as left coronary artery $(L C A)$ when the anomalous coronary was the left coronary from the right sinus of Valsalva. SCD, Sudden cardiac death; VSD, ventricular septal defect; ECMO, extracorporeal membrane oxygenation.

otherwise structurally normal hearts; 1 had isolated ventricular ectopy that was considered to be benign on the basis of exercise testing and persists after surgical unroofing.

\section{Diagnosis}

All 26 patients who were initially seen at Children's Hospital of Wisconsin had the diagnosis made by TTE. One patient with SCD and electrocardiographic evidence of myocardial injury was transferred to Children's Hospital after cardiac catheterization at the outside facility detected AAOCA. Diagnosis by TTE was usually made from a short-axis view in the plane of the aortic root at the level of the coronary artery origins. AAOCA was initially suspected on the basis of 2-dimensional imaging because a vessel was seen running within the anterior aortic wall. Color Doppler flow mapping confirmed the presence of an anomalous coronary as a linear diastolic flow signal coursing toward the appropriate sinus within the anterior aortic wall (Figure 2). Most patients (17/26) underwent no additional preoperative imaging studies after TTE diagnosis other than intraoperative transesophageal echocardiography, and most of those additional studies were performed early in our experience (Table 1). All patients (except those who were seen after SCD) underwent exercise testing before surgery, and none had evidence of inducible myocardial ischemia or ectopy.

\section{SURGICAL FINDINGS}

The coronary origin and intramural segment of the anomalous coronary was confirmed by direct visual inspection after aortotomy in all suspected cases. All patients had 2 separate coronary orifices arising from the same sinus with a variable intramural course within the anterior aortic wall. In the 7 patients who had a left AAOCA, all had a long intramural course within the right sinus, but only 1 of the 7 had a slitlike left coronary orifice described at surgical inspection (in the patient with left AAOCA and exerciseinduced syncope). In the 20 patients who had a right AAOCA, all had a shorter intramural course within the left sinus with the coronary orifice near the commissure between the right and left sinuses. Eleven of the 20 patients with a right AAOCA were described as having a slitlike orifice at surgical inspection; of those 11, no symptoms were present in 7 at presentation (Table 1).

The surgical technique has been previously described. ${ }^{9}$ The intramural segment was unroofed by excising the common wall until the coronary exited the aorta from the appropriate sinus, and the intima of the shared wall was then sutured at the neoorifice to prevent dissection. Four patients had resuspension of the affected commissure early in the experience, when the entire intramural segment was resected; subsequently, commissural disruption was avoided by resecting only the intramural wall within the appropriate sinus adjacent to the commissure without cutting across it. All patients had good biventricular function according to transesophageal echocardiography at completion of surgery after separation from cardiopulmonary bypass. In each case, an unobstructed orifice in the appropriate sinus could be visualized by imaging and color Doppler flow. 


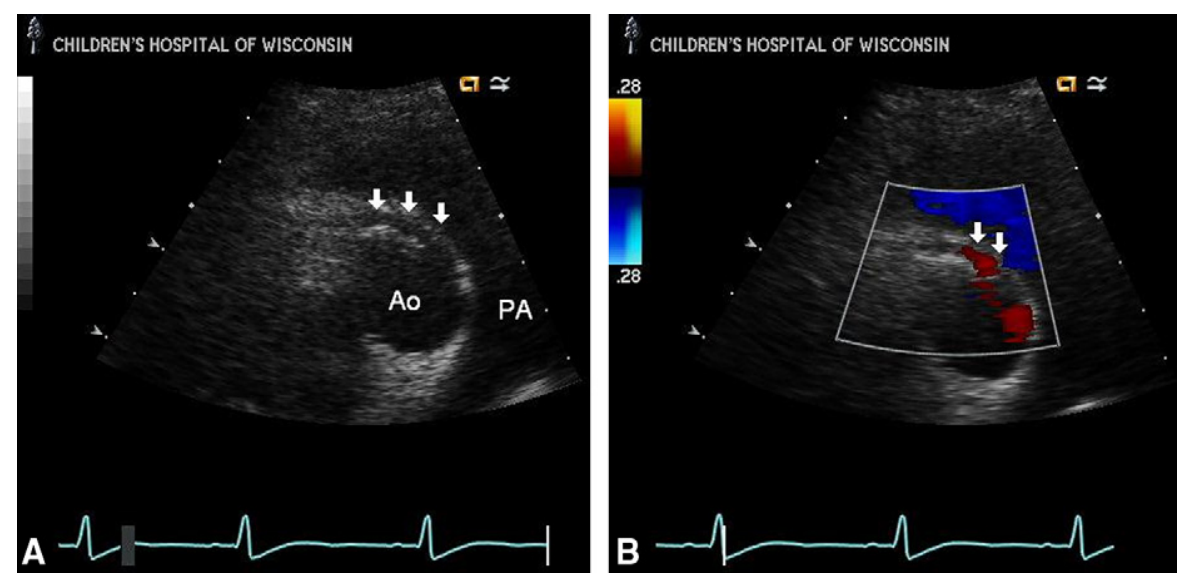

FIGURE 2. Two-dimensional echocardiographic images from a short-axis view in a patient with anomalous origin of the right coronary artery from the left sinus of Valsalva and an intramural course of the anomalous coronary. A, The anomalous left coronary artery can be seen arising more posteriorly and leftward from the left sinus of Valsalva and coursing intramural within the anterior aortic wall (arrows) between the aorta (Ao) and the pulmonary artery ( $P A$ ) toward the left sinus of Valsalva. B, Color Doppler interrogation helps confirm the diagnosis, with a linear red diastolic Doppler signal seen in the intramural segment as flow moves anteriorly toward the right sinus from its origin in the left sinus.

\section{Surgical Outcome}

Hospital stay was typically brief and uncomplicated, except in the case of the 3 patients who had aborted SCD; the other 24 patients had an average stay of $3.7 \pm 0.86$ days (range, 3-6 days). The 3 patients with SCD had stays of 9,24 , and 90 days, with extended care needed for neurologic complications related to the SCD episode. There have been no deaths in the group, and all patients are symptom free at a mean follow-up interval of 1.8 years (range, 1 week-8.2 years), with laminar flow into the proximal coronary according to serial postoperative TTE and normal ventricular function. Of the 4 patients who underwent commissure resuspension after unroofing, aortic insufficiency is trivial in 3 and absent in 1 according to TTE at current follow-up. Four additional patients have trivial aortic insufficiency that was identified after surgery. Nineteen of the 27 patients have undergone postoperative exercise testing at our institution without evidence of myocardial ischemia; no differences between the preoperative and postoperative exercise studies were found. No patient who underwent the unroofing procedure is currently restricted from activities, and none have had symptoms of myocardial ischemia or syncope.

\section{DISCUSSION}

Our cohort encompasses the largest single-center experience of surgical unroofing repair for AAOCA in children reported to date, and the early outcome results are encouraging and consistent with previous reports. There is little consensus regarding the best approach to these patients, but our results again document that the procedure can be performed safely and effectively to create a patent neoorifice in the correct sinus without bypass grafting, coronary reimplantation, patch angioplasty, or pulmonary ar- tery translocation. We observed no cases of recurrent or new myocardial ischemia after the unroofing procedure, and no significant aortic insufficiency related to commissure takedown or the unroofing has developed. A single study had previously reported subclinical and inconsistently reproducible evidence suggestive of ischemia after surgical unroofing (with lack of consistent findings in any single patient according to exercise stress testing, stress echocardiography, and myocardial perfusion scanning). ${ }^{12}$ We found no evidence of this in our cohort and have placed no activity restrictions after surgery. In light of the prospect of lifelong activity restriction when AAOCA is identified (as currently recommended by American Heart Association and American College of Cardiology guidelines ${ }^{13}$ ) if surgery is not performed, these early results demonstrate that unroofing not only may alleviate the risk of SCD but may also help avoid a medically mandated lifetime of inactivity, with the well-substantiated increased risk of metabolic syndrome and premature acquired coronary artery disease. No clear best practice has been established, although both establishment of a national registry of AAOCA and development of prospective clinical trials are being pursued.

AAOCA is a rare but important cause of SCD in children and adolescents, particularly with exercise. In a previous case series, the incidence of SCD in young athletes secondary to congenital coronary anomalies was estimated at 0.15 to 0.32 events $/ 100,000$ athletes per year. Congenital coronary anomaly was the 5th most common cause of SCD behind cardiomyopathies, acquired coronary artery disease, cardiac conduction disease, and myocarditis. ${ }^{14}$ The cardiovascular causes posing the highest risk of sports-related SCD were AAOCA (relative risk, 79), arrhythmogenic ventricular cardiomyopathy (relative risk, 5.4), and premature coronary artery disease (relative risk, 2.6). The incidence 
was not influenced by a universal preparticipation screening policy for sports that included focused history, physical examination, and 12-lead electrocardiography. ${ }^{15}$

As demonstrated in our series, TTE can be an effective and reliable tool in the accurate identification of these lifethreatening coronary artery abnormalities, and assessment of coronary origins should be part of the standard imaging protocol for all echocardiographic labs with targeted views, multiple frequency transducers, and focused color Doppler interrogation of the anterior aortic wall. ${ }^{7,9}$ The echocardiographic diagnosis was correlated with intraoperative findings in all cases in this series; that is, the intramural course predicted by TTE was always correct so that the planned surgical unroofing was always the procedure performed. No reimplantations or other coronary manipulations were necessary. Although an intramyocardial course of AAOCA has been described in which the anomalous coronary runs within the myocardial sulcus between the great arteries rather than intramurally within the aortic wall, ${ }^{16}$ we have had no patients with this lesion referred for surgical intervention at Children's Hospital of Wisconsin. Other imaging modalities, such as coronary angiography, computed tomography, ${ }^{17-19}$ and magnetic resonance imaging ${ }^{20}$ are also effective for delineation of coronary artery anatomy and can be used to provide diagnostic coronary imaging. As shown again in our series, exercise testing appears to add little value in risk stratification after AAOCA has been prospectively identified, even for those with symptoms of cardiac ischemia. ${ }^{9,21}$ Symptoms preceding SCD are described commonly in all autopsy series that identify AAOCA as the cause of death, a finding that emphasizes the need for clear delineation of coronary anatomy in patients with symptoms suggestive of myocardial ischemia.

The surgical findings in our series are compelling and suggest that symptoms alone may not allow effective risk stratification of patients. Only 1 of the 7 patients with left AAOCA had a slitlike anomalous coronary ostium at surgical inspection, despite the fact the 4 of the 7 had symptoms consistent with exercise-induced ischemia (and 3 actually were seen after aborted SCD). In contrast, 11 of 20 patients with right AAOCA had a slitlike orifice at inspection. and 7 of those 11 had no symptoms at presentation. It appears that the etiology of ischemia with this lesion is multifactorial and not always explained by a slitlike ostium or the acute angle of origin of the anomalous coronary from the aorta that may alter flow patterns into that coronary artery bed. ${ }^{22}$ Intravascular ultrasonography in AAOCA has documented both coronary hypoplasia and localized systolic lateral compression of the intramural segment of anomalous coronaries that run within the aortic wall. Not surprisingly, this degree of compression appears to have individual variations that probably explain the unpredictable response to exercise in this patient group. ${ }^{23,24}$ The coronary narrowing can be exacerbated by pharmacologic challenge that likely mimics exercise conditions. In addition, the length of the intramural segment may play a role in the development of ischemia, because longer segments may accentuate the degree of stenosis caused by luminal distortion during exercise. We observed that left AAOCA was associated with a consistently longer intramural segment in our patients (both echocardiographically and at surgery) than seen in those with right AAOCA. This observation correlates with the apparent increased risk of SCD described for left AAOCA in almost all series that have reviewed autopsy findings of SCD in children and young adults.

Importantly, the patients in our cohort with left AAOCA and aborted SCD did not stabilize after initial resuscitation, despite evidence of laminar coronary flow in the anomalous intramural segment according to echocardiography. The etiology of ongoing ectopy and myocardial dysfunction after resuscitation in these patients is unclear, ${ }^{25}$ emphasizing how little we understand about the mechanisms of ischemia and how critical immediate repair of the AAOCA is in establishing safe myocardial perfusion when SCD is the presenting symptom. Finally, risk is almost certainly influenced by the precipitating conditions (usually vigorous exercise $^{2-4,21}$ ) at the time of the ischemic insult, as well as the location and amount of myocardium supplied by the anomalous coronary. Unfortunately, there is currently no effective technique for quantifying these anatomic and physiologic risks. Because of this, we have adopted a strategy of surgical unroofing in all patients identified with the intramural form of AAOCA, irrespective of symptoms.

\section{CONCLUSIONS}

AAOCA is frequently characterized by an intramural course, which can be reliably identified by TTE with rigorous 2-dimensional and color Doppler protocols for coronary imaging. The intramural form can be safely repaired by unroofing the intramural segment without early morbidity or mortality. Symptoms of possible ischemia are common but do not always correlate with coronary ostial findings at surgery. Patients with AAOCA and resuscitated SCD should be considered to represent a surgical emergency and should undergo unroofing immediately on presentation.

\section{References}

1. Liberthson RR, Dinsmore RE, Fallon JT. Aberrant coronary artery origin from the aorta. Report of 18 patients, review of literature and delineation of natural history and management. Circulation. 1979;59:748-54.

2. Frescura C, Basso C, Thiene G, Corrado D, Pennelli T, Angelini A, et al. Anomalous origin of coronary arteries and risk of sudden death: a study based on an autopsy population of congenital heart disease. Hum Pathol. 1998;29:689-95.

3. Taylor AJ, Rogan KM, Virmani R. Sudden cardiac death associated with isolated congenital coronary artery anomalies. J Am Coll Cardiol. 1992;20:640-7.

4. Kragel AH, Roberts WC. Anomalous origin of either the right or left main coronary artery from the aorta with subsequent coursing between aorta and pulmonary trunk: analysis of 32 necropsy cases. Am J Cardiol. 1988;62:771-7. 
5. Eckart RE, Jones SO IV, Shry EA, Garrett PD, Scoville SL. Sudden death associated with anomalous coronary origin and obstructive coronary disease in the young. Cardiol Rev. 2006;14:161-3.

6. Frommelt PC, Friedberg DZ, Frommelt MA, Williamson JG. Anomalous origin of the right coronary artery from the left sinus of Valsalva: transthoracic echocardiographic diagnosis. J Am Soc Echocardiogr. 1999;12:221-4.

7. Frommelt PC, Berger S, Pelech AN, Bergstrom S, Williamson JG. Prospective identification of anomalous origin of left coronary artery from the right sinus of Valsalva using transthoracic echocardiography: importance of color Doppler flow mapping. Pediatr Cardiol. 2001;22:327-32.

8. Maron BJ, Leon MB, Swain JA, Cannon RO 3rd, Pelliccia A. Prospective identification by two-dimensional echocardiography of anomalous origin of the left main coronary artery from the right sinus of Valsalva. Am J Cardiol. 1991;68: $140-2$.

9. Frommelt PC, Frommelt MA, Tweddell JS, Jaquiss RD. Prospective echocardiographic diagnosis and surgical repair of anomalous origin of a coronary artery from the opposite sinus with an interarterial course. J Am Coll Cardiol. 2003; 42:148-54.

10. Mustafa I, Gula G, Radley-Smith R, Durrer S, Yacoub M. Anomalous origin of the left coronary artery from the anterior aortic sinus: a potential cause of sudden death. Anatomic characterization and surgical treatment. J Thorac Cardiovasc Surg. 1981;82:297-300.

11. Romp RL, Herlong R, Landolfo CK, Sanders SP, Miller CE, Ungerleider RM, et al. Outcome of unroofing procedure for repair of anomalous aortic origin of left or right coronary artery. Ann Thorac Surg. 2003;76:589-96.

12. Brothers JA, McBride MG, Seliem MA, Marino BS, Tomlinson RS, Pampaloni MH, et al. Evaluation of myocardial ischemia after surgical repair of anomalous aortic origin of a coronary artery in a series of pediatric patients. J Am Coll Cardiol. 2007;50:2078-82.

13. Graham TP Jr, Driscoll DJ, Gersony WM, Newburger JW, Rocchini A, Towbin JA. Task Force 2: congenital heart disease. J Am Coll Cardiol. 2005; 45:1326-33.

14. Corrado D, Basso C, Schiavon M, Thiene G. Does sports activity enhance the risk of sudden cardiac death? J Cardiovasc Med (Hagerstown). 2006;7: 228-33.
15. Corrado D, Basso C, Pavei A, Michieli P, Schiavon M, Thiene G. Trends in sudden cardiovascular death in young competitive athletes after implementation of a preparticipation screening program. JAMA. 2006;296:1593-601.

16. Frommelt MA, Frommelt PC. Vascular anomalies. In: Eidem BW, Cetta F, O'Leary PW, eds. Echocardiography in pediatric and adult congenital heart disease. Philadelphia: Wolters Kluwer; 2010. p. 347-8.

17. de Jonge GJ, van Ooijen PM, Piers LH, Dikkers R, Tio RA, Willems TP, et al. Visualization of anomalous coronary arteries on dual-source computed tomography. Eur Radiol. 2008 Nov;18:2425-32.

18. Komatsu S, Sato Y, Ichikawa M, Kunimasa T, Ito S, Takagi T, et al. Anomalous coronary arteries in adults detected by multislice computed tomography: presentation of cases from multicenter registry and review of the literature. Heart Ves sels. 2008;23:26-34

19. Kacmaz F, Ozbulbul NI, Alyan O, Maden O, Demir AD, Balbay Y, et al. Imaging of coronary artery anomalies: the role of multidetector computed tomography. Coron Artery Dis. 2008;19:203-9.

20. Su JT, Chung T, Muthupillai R, Pignatelli RH, Kung GC, Diaz LK, et al. Usefulness of real-time navigator magnetic resonance imaging for evaluating coronary artery origins in pediatric patients. Am J Cardiol. 2005;95:679-82.

21. Basso C, Maron BJ, Corrado D, Thiene G. Clinical profile of congenital coronary artery anomalies with origin from the wrong sinus leading to sudden death in competitive athletes. J Am Coll Cardiol. 2000;35:1493-501.

22. Angelini P. Coronary artery anomalies: an entity in search of an identity. Circu lation. 2007;115:1296-305.

23. Dimopoulos K, Di Mario C, Barlis P, Pennell D, Goktekin O, Kaddoura S, et al Haemodynamic significance of an anomalous right coronary with inter-arterial course assessed with intracoronary pressure measurements during dobutamine challenge. Int J Cardiol. 2008;126:e32-5.

24. Tsujita K, Maehara A, Mintz GS, Franklin-Bond T, Mehran R, Stone GW, et al. In vivo intravascular ultrasonic assessment of anomalous right coronary artery arising from left coronary sinus. Am J Cardiol. 2009;103:747-51.

25. Saeed M, Gabara R, Strasberg B, Kusniec J, Rosanio S, Ware DL, et al. Reperfusion-related polymorphic ventricular tachycardia as a possible mechanism of sudden death in patients with anomalous coronary arteries. Am J Med Sci. 2005;329:327-9. 